\title{
Terapias alternativas na odontologia: ação antibiofilme do extrato de Curcuma longa associado ou não a Terapia Fotodinâmica contra Candida spp.
}

\author{
Alternative therapies in dentistry: antibiofilm action of Curcuma longa extract associated or not \\ with Photodynamic Therapy against Candida spp. \\ Terapias alternativas en odontología: acción antibiofilm del extracto de Curcuma longa asociada o \\ no a la Terapia Fotodinámica contra Candida spp.
}

\section{Resumo}

Na odontologia, a candidose bucal é causada principalmente por Candida albicans mas espécies não-albicans também estão emergindo e a resistência aos antifúngicos é uma crescente. As terapias alternativas como a fitoterapia e a terapia fotodinâmica (TFD) são promissoras nesse sentido. O objetivo do estudo foi avaliar a ação do extrato glicólico de Curcuma longa associado ou não com luz sobre biofilme de Candida albicans, Candida krusei e Candida glabrata. Biofilmes monomicrobianos das espécies de Candida foram formados por $48 \mathrm{~h}$, seguido do tratamento com

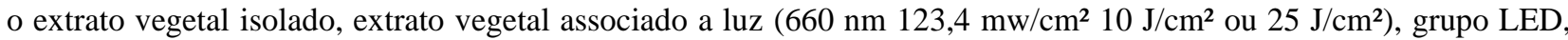
grupo salina e nistatina. A viabilidade celular foi analisada por meio do teste de MTT e as densidades ópticas transformadas em percentual de atividade metabólica. Para as analises estatísticas, se os dados atenderam os pressupostos foram analisados por ANOVA e Teste de Tukey, caso contrário, pelo teste de Kruskal-Wallis e Dunn, considerando nível de significância de 5\%. O extrato de C. longa aplicado ou não a TFDa promoveu significativa ação antibiofilme sobre as espécies de Candida analisadas ( $\mathrm{p}<0,05)$, sendo que o maior percentual de redução foi encontrado sobre $C$. krusei, com reduções acima de $45 \%$. Em conclusão, o extrato de $C$. longa associado ou não TFDa apresentou ação antibiofilme contra Candida spp. e sua atividade antifúngica foi igual e até superior a nistatina sobre algumas espécies. O extrato de $C$. longa revelou-se um potente antifúngico para ser explorado dentro das terapias alternativas na odontologia

Palavras-chave: Biofilme; Candida; Curcuma longa; Fitoterapia; Fotoquimioterapia.

\footnotetext{
Abstract

In dentistry, oral candidiasis is mainly caused by Candida albicans but non-albicans species are also emerging and antifungal resistance is increasing. Alternative therapies such as herbal medicine and photodynamic therapy (PDT) are promising in this regard. The study aimed to evaluate the action of Curcuma longa glycolic extract associated or not with light on Candida albicans, Candida krusei, and Candida glabrata biofilms. Monomicrobial biofilms of Candida
} 
species were formed for $48 \mathrm{~h}$, followed by treatment with the isolated plant extract, plant extract associated with light $\left(660 \mathrm{~nm} 123.4 \mathrm{MW} / \mathrm{cm}^{2} 10 \mathrm{~J} / \mathrm{cm}^{2}\right.$ or $\left.25 \mathrm{~J} / \mathrm{cm}^{2}\right)$, group LED, saline and nystatin group. Cell viability was analyzed using the MTT test and and optical densities were transformed into percentage of metabolic activity. For statistical analysis, if the data met the assumptions, they were analyzed by ANOVA and Tukey's test, otherwise, by the KruskalWallis test and Dunn's, considering a significance level of 5\%. The C. longa extract applied or not to PDTa promoted significant antibiofilm action on the Candida species analyzed ( $<<0.05)$, and the highest percentage of reduction was found on $C$. krusei, with reductions above $45 \%$. In conclusion, the $C$. longa extract associated or not with PDTa showed antibiofilm action against Candida spp. and its antifungal activity was equal and even superior to that of nystatin on some species. The extract of $C$. longa proved to be a potent antifungal to be explored within alternative therapies in dentistry.

Keywords: Biofilm; Candida; Curcuma longa; Phytotherapy; Photochemotherapy.

\section{Resumen}

En odontología, la candidiasis oral está causada principalmente por Candida albicans, pero también están surgiendo especies no albicans y la resistencia a los antifúngicos va en aumento. Las terapias alternativas como la medicina herbaria y la terapia fotodinámica son prometedoras en este sentido. El objetivo del estudio fue evaluar la acción del extracto glicólico de Curcuma longa asociado o no con luz sobre biopelículas de Candida albicans, Candida krusei y Candida glabrata. Se formaron biopelículas monomicrobianas de especies de Candida durante $48 \mathrm{~h}$, seguido de un

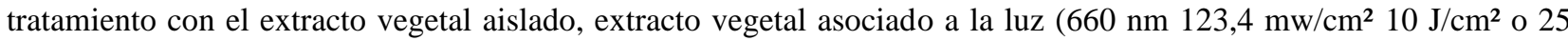
$\mathrm{J} / \mathrm{cm}^{2}$ ), grupo LED, grupo de solución salina y nistatina. La viabilidad celular se analizó mediante la prueba MTT y densidades ópticas transformadas en porcentaje de actividad metabólica. Para el análisis estadístico, si los datos cumplieron con los supuestos, fueron analizados por ANOVA y la prueba de Tukey, en caso contrario, por la prueba de Kruskal-Wallis y Dunn, considerando un nivel de significación del 5\%. El extracto de C. longa aplicado o no a TFDa promovió una acción antibiofilm significativa sobre las especies de Candida analizadas (p < 0,05), y el mayor porcentaje de reducción se encontró en $C$. krusei, con reducciones por encima de $45 \%$ En conclusión, el extracto de C. longa asociado o no con TFDa mostró acción antibiopelícula contra Candida spp y su actividad antifúngica fue igual e incluso superior a la de la nistatina en algunas especies. El extracto de $C$. longa demostró ser un potente antifúngico a ser explorado dentro de las terapias alternativas en odontología.

Palabras clave: Biopelícula; Candida. Curcuma longa; Fitoterapia; Fotoquimioterapia.

\section{Introdução}

Espécies de Candida são encontradas na microbiota oral do hospedeiro convivendo em associação com os demais microrganismos existentes. Todavia, Candida spp. são fungos oportunistas e quando há um desequilíbrio no meio, podem causar a candidose bucal. Os pacientes mais acometidos por esta infecção são os diabéticos, idosos, usuários de próteses dentárias e imunocomprometidos (Guandalini Cunha et al., 2020). Devido a pandemia do novo coronavírus, verifica-se alguns relatos na literatura os quais descrevem que pacientes com COVID-19 e com estomatite protética (causada por Candida spp.) que necessitam de ventilação mecânica, podem ter o quadro clínico agravado e com isso, ocorre um aumento da morbidade e mortalidade desses pacientes (Jerônimo et al., 2021). A formação de biofilme é um importante fator de virulência para uma série de espécies de Candida já que confere resistência significativa aos antifúngicos (Kawsud et al., 2014). Candida albicans representa mais de $80 \%$ dos isolados clínicos, porém a literatura demonstra que a epidemiologia das infecções por Candida têm sofrido modificações com o surgimento de espécies não-albicans ( Pfaller et al., 2010; Michael \& Pfaller et al., 2011; Salerno et al., 2011).

A resistência aos medicamentos antifúngicos tem sido cada vez mais comum. A literatura já relata que Candida glabrata e Candida krusei apresentam resistência intrínseca aos ázois (Morio et al., 2017). Pesquisas mostram que na última década, as infecções causadas por Candida spp. estão crescendo devido à alta resistência antifúngica e as reações indesejadas que os medicamentos provocam no organismo dos pacientes ( Vieira et al., 2019). No atual cenário da pandemia da COVID19, o emprego dos corticosteroides têm sido uma realidade. Contudo, sabe-se que tais medicamentos apresentam efeito imunossupressor sobre neutrófilos, monócitos e macrófagos e tal situação somada à resistência antimicrobiana predispõe os pacientes à candidíase invasiva (Arastehfar et al., 2020).

Nos últimos anos, muitos estudos estão sendo direcionados na busca por novas alternativas de tratamentos que 
sejam efetivos contra as infecções resistentes e biocompatíveis. Os fitoterápicos surgem como uma alternativa para controlar infecções bucais. Relatos na literatura comprovam as diversas atividades biológicas da Curcuma longa L., popularmente conhecido como açafrão-da-terra (Sueth-Santiago et al., 2015). Um deles é a evidência do potencial benéfico da curcumina, o fitoquímico isolado da C. longa, no controle do biofilme dentário ( $\mathrm{Li}$ et al., 2019). Evidências promissoras dos efeitos terapêuticos do extrato de açafrão javanês (outro rizoma do gênero Curcuma), comprovam o potencial inibitório contra biofilme de C. albicans quando em fase de maturação (Puspitawati et al., 2019). Em outro estudo, C. longa esteve entre os fitoterápicos que apresentaram o melhor efeito inibitório com potencial antifúngico sobre cepas clínicas de $C$. albicans (Samadi et al., 2019). De maneira geral, a ação anti-Candida da C. longa é bem relatada na literatura (Cheraghipour et al., 2020).

A fotoquimioterapia ou terapia fotodinâmica antimicrobiana (TFDa - do inglês antimicrobial photodynamic therapy) consiste na administração de um fotossensibilizador (Fs) e de irradiação de luz em um comprimento de onda específico, o que produz espécies reativas de oxigênio (ERO) causando efeito citotóxico nas células alvo. Pela literatura, sabese que os Fs podem ser localizados em moléculas de plantas (Bruschi et al., 2019). A TFDa tem sido descrita como "uma terapia que dificilmente promoverá resistência antimicrobiana" devido ao seu mecanismo de ação, uma vez que o oxigênio e os radicais livres interagem com diferentes estruturas da célula alvo (Wainwright et al., 2017).

O extrato alcóolico de $C$. longa aplicado como Fs na TFDa sobre periodontopatógenos promoveu efeito antimicrobiano similar a clorexidina $0,12 \%$ (Saitawee et al., 2018). A TFDa mediada por curcumina $\left(450 \mathrm{~nm}, 67 \mathrm{~mW} / \mathrm{cm}^{2} \mathrm{ou}\right.$ $20.1 \mathrm{~J} / \mathrm{cm}^{2}$ ) analisada sobre biofilme de C. albicans promoveu evidente redução na contagem de Unidades Formadoras de Colônias/mL (da Silva et al., 2019). Os estudos citados utilizam a C. longa no comprimento de onda de luz azul, contudo o uso do LED vermelho com o extrato de C. longa como Fs foi descrito na literatura por Nardini et al. (2019). Os autores testaram diferentes bandas de absorção de luz do espectro visível: violeta (405 nm), azul (460 nm) e vermelho (660 nm). A TFDa mediada por $C$. longa produziu redução microbiana significativa em culturas planctônicas e biofilme de Enterococcus faecalis. Quando o composto foi ativado na luz vermelha $(660 \mathrm{~nm})$, C. longa mostrou produção de ERO semelhante ao azul de metileno que é considerado padrão ouro para TFDa com uma fonte de luz vermelha (Nardini et al., 2019).

A Luz na faixa do vermelho possui comprimento de onda de 610-760 nm e é utilizada para acelerar o processo de cicatrização de feridas, possui poder de analgesia e ação anti-inflamatória. O uso do LASER vermelho tem sua eficácia comprovada para tais finalidades terapêuticas e o emprego do LED vermelho é mais recente, porém apresenta resultados tão bem-sucedidos quanto o LASER e os benefícios da aplicação de LED vermelho no tecido mole são inúmeros (A. B. H. Vieira, 2020). O fracionamento da dose de luz no momento da irradiação tem se apresentado como um protocolo vantajoso por permitir a reoxigenação do tecido durante o período em que não está sendo iluminado, assim potencializando o efeito da terapia fotodinâmica (Sampaio et al., 2020). Diante dessas duas alternativas terapêuticas promissoras, a fitoterapia e a TFDa, o presente estudou avaliou a ação do extrato glicólico de $C$. longa associado ou não com luz no comprimento de $660 \mathrm{~nm}$ (faixa do vermelho) com protocolo de luz fracionada (10 ou $\left.25 \mathrm{~J} / \mathrm{cm}^{2} ; 123,4 \mathrm{mw} / \mathrm{cm}^{2}\right)$ sobre biofilme de C. albicans, C. krusei e $C$. glabrata.

\section{Metodologia}

A metodologia do presente estudo foi baseada no estudo de Nardini et al. (2019) com modificações. O extrato glicólico de $C$. longa L. foi adquirido na empresa Seiva Brazilis, SP na concentração de 200mg/mL. Foi utilizado uma Biotable com 48 LEDs, potência de 3W por LED, no comprimento de onda de $660 \mathrm{~nm}$ (Biopdi/Irrad-Led) e cepas de Candida de referência (ATCC - American Type Culture Collection) de C. albicans (ATCC 18804), C. glabrata (ATCC 9030), C. krusei (ATCC 6258) pertencentes ao Laboratório de Microbiologia e Imunologia do Instituto de Ciência e Tecnologia da UNESP. 


\subsection{Aplicação das terapias sobre biofilmes de Candida spp.}

Candida spp. foram cultivadas $\left(37^{\circ} \mathrm{C} / 24 \mathrm{~h}\right)$ em ágar Sabouraud-dextrose (SD - Himedia, Mumbai, Índia) em seguida, foram realizadas as preparações das suspensões microbianas onde colônias das respectivas espécies de Candida foram diluídas em solução salina estéril $(\mathrm{NaCl}$ 0,9\%) e homogeneizado em Vortex por $10 \mathrm{~s}$. foi realizada a padronização em espectrofotômetro, para obtenção de $10^{7} \mathrm{UFC} / \mathrm{mL}$. Posteriormente, foram adicionados em microplacas $200 \mu \mathrm{L} /$ poço da suspensão ajustada de Candida, que foi levada para incubação $\left(37^{\circ} \mathrm{C} / 90 \mathrm{~min}\right)$ para a adesão inicial das células fúngicas ao poço. Em seguida, o sobrenadante foi descartado e foi adicionado caldo BHI (Brain Heart Infusion - Kasvi). A incubação, seguiu por $48 \mathrm{~h}$ para formação do biofilme, com substituição do caldo após 24 h de incubação. Após a formação do biofilme, o sobrenadante dos poços foi descartado e os poços correspondentes aos grupos somente extrato vegetal e TFDa receberam o extrato de C. longa $(100 \mathrm{mg} / \mathrm{mL}$ ) e os poços dos grupos controle negativo (solução salina) e somente irradiado LED receberam solução salina para incubação por 20 min no escuro, sendo $n=10$. Em seguida, os grupos LED e TFDa foram irradiados $\left(1^{\circ}\right.$ protocolo de irradiação: $123,4 \mathrm{mw} / \mathrm{cm}^{2} 10 \mathrm{~J} / \mathrm{cm}^{2}$ por $81 \mathrm{~s}$ ou $2^{\circ}$ protocolo de irradiação: $123,4 \mathrm{mw} / \mathrm{cm}^{2} 25 \mathrm{~J} / \mathrm{cm}^{2}$ por $203 \mathrm{~s}$ ) com a luz fracionada. Os grupos controle negativo e os que receberam somente o extrato, foram mantidos no escuro pelo mesmo período de irradiação. Ainda, um grupo foi tratado com nistatina (suspensão oral $100.000 \mathrm{UI} / \mathrm{mL}$ ) para fins de comparação dos efeitos. Após exposição ao tratamento, os poços foram lavados com solução salina e a mesma descartada, para eliminar células não aderentes que sofreram com a ação da terapia. Foi realizado o teste de viabilidade celular das células fúngicas no qual foram adicionados $100 \mu \mathrm{L}$ da solução de MTT (3-(4,5-Dimethylthiazol-2-yl)-2,5- Diphenyltetrazolium Bromide) em cada poço e as placas foram incubadas ao abrigo da luz a $37{ }^{\circ} \mathrm{C}$ durante $1 \mathrm{~h}$. Passado o período de incubação a solução de MTT retirada seguido pela adição de $100 \mu \mathrm{L}$ de Dimetilsufóxido (DMSO). As placas foram novamente incubadas em estufa a $37^{\circ} \mathrm{C}$ por 10 minutos e colocadas no Shaker sob agitação constante por 10 minutos. Em seguida foram obtida as densidades ópticas (DO) por meio de uma leitora de microplaca em $570 \mathrm{~nm}$ e as DO obtidas serão convertidas em percentual de atividade metabólica das células fúngicas.

\subsection{Análise estatística}

Todos os dados foram inicialmente, avaliados quanto a normalidade por meio dos testes: Shapiro-Wilk, Kolmogorov-Smirnov e D'Agostino \& Pearson omnibus e quanto a homogeneidade por meio do software Bioestat 5.0. Para seguir com as análises estatísticas, se os dados apresentaram normalidade e homogeneidade foram analisados por ANOVA e Teste de Tukey, caso contrário, pelo teste de Kruskal-Wallis e Teste de Dunn. Foi utilizado o programa GraphPad Prism 5.0, considerando-se nível de significância de 5\%.

\section{Resultados}

\subsection{Sobre C. albicans}

Pode-se observar na figura 1A que o grupo tratado com extrato de $C$. longa e o grupo TFDa com extrato $\left(10 \mathrm{~J} / \mathrm{cm}^{2}\right)$ produziram reduções significativas e diferiram estatisticamente do controle negativo (solução salina) sendo $\mathrm{p}<0,05$. No $2^{\circ}$ protocolo de irradiância com dose de $25 \mathrm{~J} / \mathrm{cm}^{2}$ (figura $1 \mathrm{~B}$ ), o grupo tratado com TFDa diferiu estatisticamente do controle negativo $(p<0,05)$, produzindo percentual de redução de $23,3 \%$ do biofilme de $C$. albicans quando comparado ao controle. Tal percentual foi maior do que a nistatina que promoveu reduções de apenas $4,8 \%$ não diferindo estatisticamente do grupo solução salina $(\mathrm{p}>0,05)$. 
Figura 1: Resultados dos tratamentos aplicando as terapias alternativas sobre biofilme de Candida albicans.

\section{Biofilme de Candida albicans}

A)

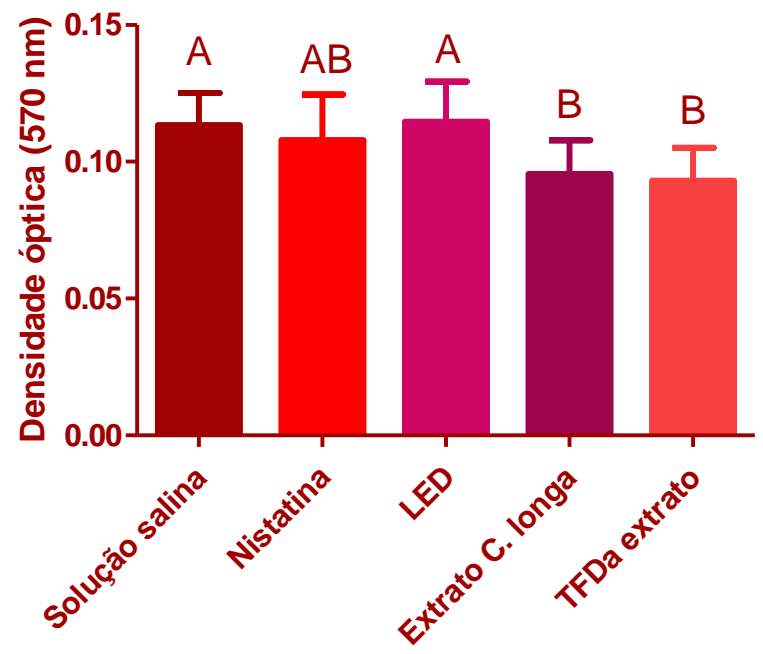

B)

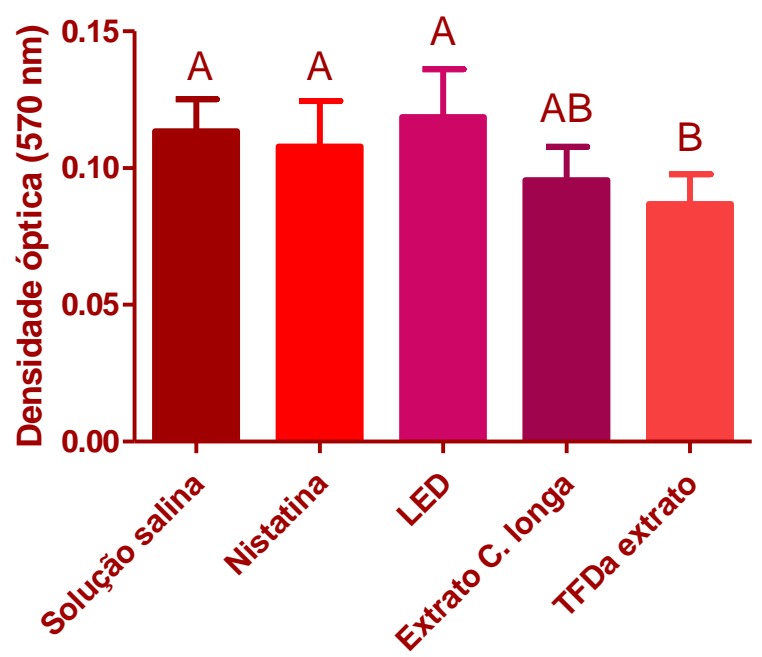

Legenda: A) grupos irradiados receberam $1^{\circ}$ protocolo de irradiação $\left(10 \mathrm{~J} / \mathrm{cm}^{2}\right)$. B) grupos irradiados receberam $2^{\circ}$ protocolo de irradiação $\left(25 \mathrm{~J} / \mathrm{cm}^{2}\right)$. Letras diferentes indicam grupos estatisticamente diferentes. Valores médios \pm desvio padrão da leitura da densidade óptica (570 $\mathrm{nm})$ do biofilme de $C$. albicans após exposição tratamentos $(\mathrm{n}=10$. ANOVA, teste de Tukey $\mathrm{p} \leq 0,05)$. Fonte: Autores.

\subsection{Sobre C. glabrata}

Em ambos protocolos de irradiância (figura 2A e 2B) a TFDa com extrato e os grupos tratados com extrato de $C$. longa isolado e nistatina foram capazes de produzir reduções significativas no biofilme de C. glabrata e diferir estatisticamente do controle negativo ( $\mathrm{p}<0,05)$. A ação do extrato vegetal aplicado ou não a TFDa produziu reduções no biofilme de até $22 \%$. O antifúngico nistatina também produziu reduções significativas (25\%) no biofilme dessa espécie. 
Figura 2: Resultados dos tratamentos aplicando as terapias alternativas sobre biofilme de Candida glabrata.

\section{Biofilme de Candida glabrata}

A)

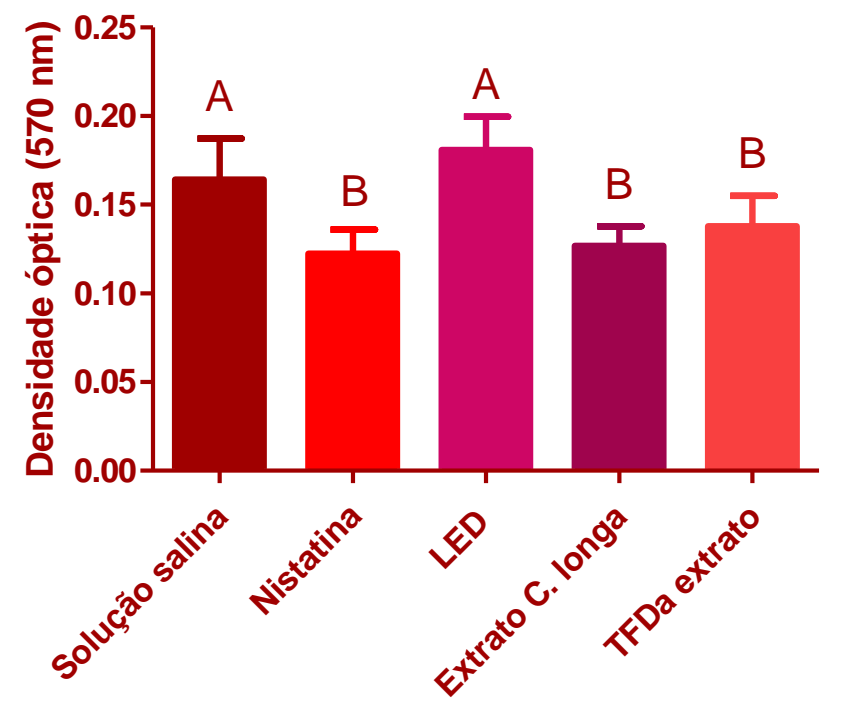

B)

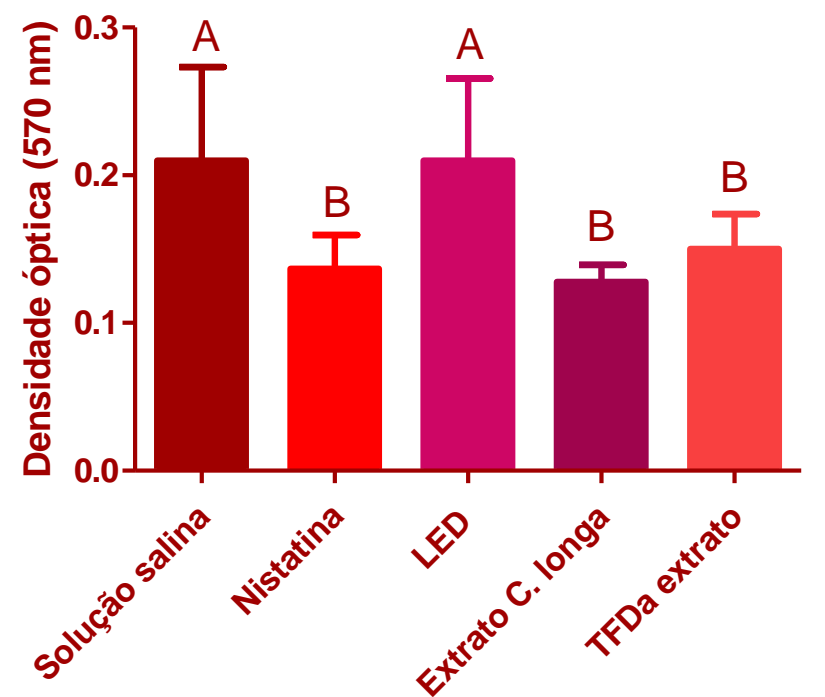

Legenda: A) grupos irradiados receberam $1^{\circ}$ protocolo de irradiação $\left(10 \mathrm{~J} / \mathrm{cm}^{2}\right)$. B) grupos irradiados receberam $2^{\circ}$ protocolo de irradiação $\left(25 \mathrm{~J} / \mathrm{cm}^{2}\right)$. Letras diferentes indicam grupos estatisticamente diferentes. Valores médios \pm desvio padrão da leitura da densidade óptica (570 $\mathrm{nm})$ do biofilme de $C$. glabrata após exposição tratamentos $(\mathrm{n}=10$. ANOVA, teste de Tukey, $\mathrm{p} \leq 0,05)$. Fonte: Autores.

\subsection{Sobre C. krusei}

Sobre C. krusei, pode-se verificar que em ambos os protocolos de irradiância (Figura 3A e 3B) a TFDa com o extrato vegetal diferiu estatisticamente do grupo salina $(\mathrm{p}<0,05)$ sendo que no $1^{\circ}$ protocolo, a TFDa com extrato produziu reduções de $47 \%$ e no $2^{\circ}$ protocolo de irradiância, de $46 \%$. Quando o extrato foi aplicado sem a luz, também produziu reduções importantes de até $49 \%$ do biofilme e diferiu estatisticamente do controle $(\mathrm{p}<0,05)$. O antifúngico nistatina também produziu reduções no biofilme, contudo, em menores proporções. 
Figura 3: Resultados dos tratamentos aplicando as terapias alternativas sobre biofilme de Candida krusei.

\section{Biofilme de Candida krusei}

A)

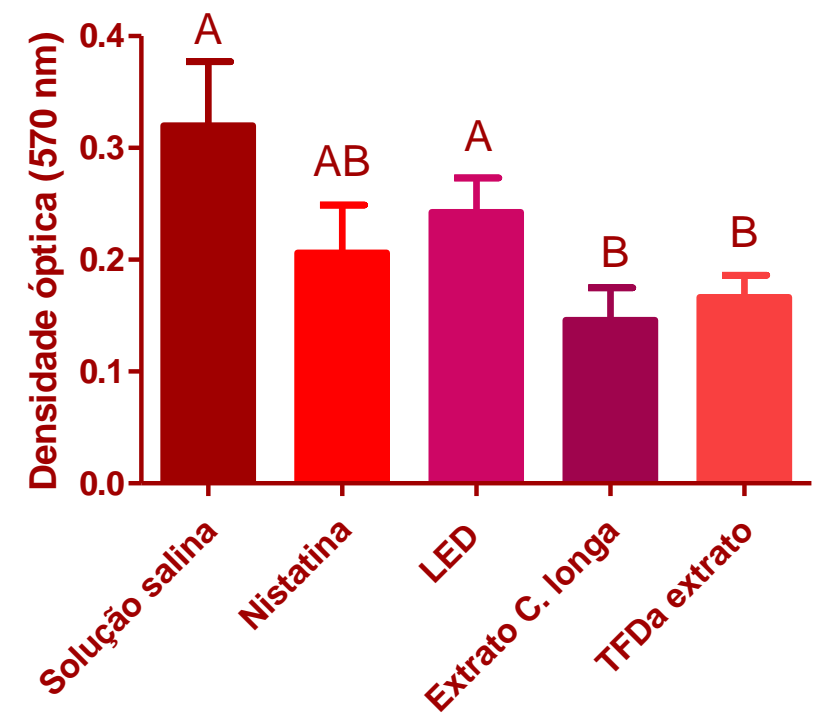

B)

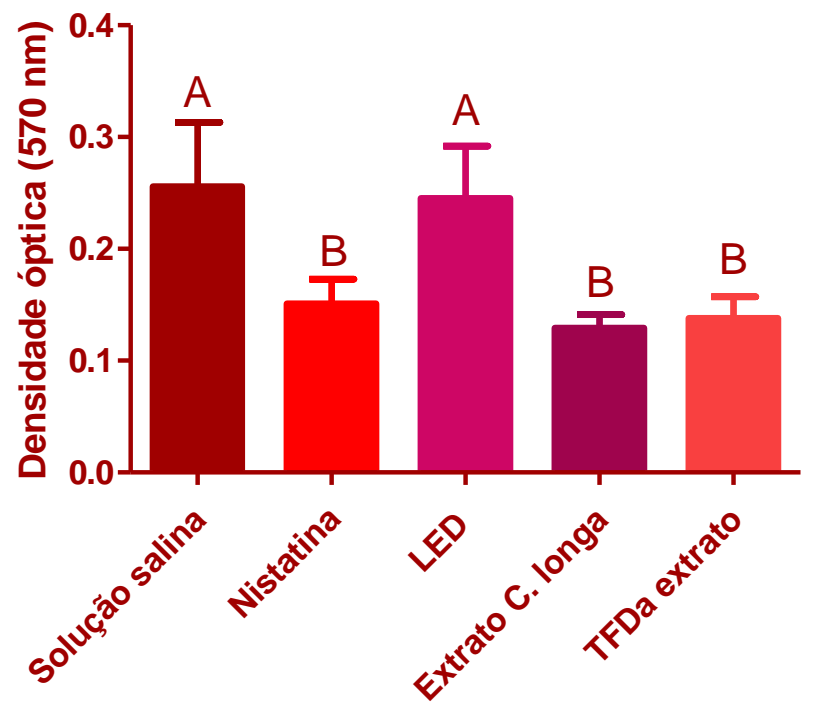

Legenda: A) grupos irradiados receberam $1^{\circ}$ protocolo de irradiação $\left(10 \mathrm{~J} / \mathrm{cm}^{2}\right)$. B) grupos irradiados receberam $2^{\circ}$ protocolo de irradiação $\left(25 \mathrm{~J} / \mathrm{cm}^{2}\right)$. Letras diferentes indicam grupos estatisticamente diferentes. Valores médios \pm desvio padrão da leitura da densidade óptica $(570$ $\mathrm{nm})$ do biofilme de $C$. krusei após exposição tratamentos $(\mathrm{n}=10$. Kruskal-Wallis, teste de Dunn $\mathrm{p} \leq 0,05)$. Fonte: Autores.

\section{Discussão}

O presente estudo avaliou o extrato glicólico de $C$. longa isolado ou aplicado na TFDa em diferentes protocolos de irradiação (660 nm; luz fracionada; 25 ou $10 \mathrm{~J} / \mathrm{cm}^{2} ; 123,4 \mathrm{mw} / \mathrm{cm}^{2}$ ). É valido ressaltar que os estudos de TFDa com C. longa também focam unicamente na aplicação dos derivados da cúrcuma na faixa de luz azul e no presente trabalho, avaliamos na faixa do vermelho, baseado no estudo de Nardini et al. (2019). Sobre biofilmes de todas as espécies de Candida analisadas, pode-se observar que o extrato de cúrcuma quando aplicado ou não a TFDa produziu reduções significativas no biofilme quando comparados ao controle negativo $(\mathrm{p}<0,05)$, principalmente quando irradiado com dose de $25 \mathrm{~J} / \mathrm{cm}^{2}$. Tais resultados estão de acordo com o estudo de Nardini e colaboradores (2019) no qual avaliaram a C. longa como Fs em diferentes bandas de absorção de luz do espectro visível: violeta $(405 \mathrm{~nm})$, azul (460 nm) e vermelho (660 nm). A TFDa mediada pelo produto vegetal produziu redução microbiana significativa em culturas planctônicas e biofilme de Enterococcus faecalis. Quando o composto foi ativado na luz vermelha $(660 \mathrm{~nm})$, C. longa mostrou produção de espécies reativas de oxigênio (ERO) semelhante ao azul de metileno que é considerado padrão ouro para TFDa com uma fonte de luz vermelha.

Candida é o patógeno mais prevalente nas infecções fúngicas que acometem o ser humano e é um dos principais contribuintes para a morte por infecção fúngica em todo o mundo (Lee et al., 2021), em especial a espécie C. albicans que apresenta resistência aos agentes fungistáticos sintéticos. Tal espécie também é frequentemente associada a cronicidade das infecções periodontais e a mais prevalente nas candidoses que acometem as mucosas orais (Monroy-Pérez et al., 2020). Em nossos achados, pode-se observar que sobre C. albicans o produto vegetal aplicado ou não a TFDa foi capaz de reduzir o biofilme de maneira mais eficiente que a nistatina, que é um dos fármacos mais utilizados nos casos de candidose oral. A 
aplicação da TFDa no $2^{\circ}$ protocolo de irradiância produziram percentuais de redução do biofilme acima de $20 \%(\mathrm{p}<0,05)$ enquanto o antifúngico foi capaz de reduzir apenas $4 \%$ do biofilme, não diferindo do grupo controle ( $p>0,05)$. Por outro lado, estudos longitudinais têm demonstrado que uma proporção considerável de pacientes está desenvolvendo infecções fúngicas por espécies de Candida não-albicans (M. A. Pfaller et al., 2010; Michael A. Pfaller et al., 2011). Em nosso estudo, o produto vegetal associado ou não a TFDa produziu reduções acima de $45 \%$ da viabilidade do biofilme de C. krusei e sobre C. glabrata, a atuação do extrato vegetal foi semelhante a nistatina, com reduções na faixa de $20 \%$ e todos os grupos que receberam o extrato vegetal diferiram estatisticamente do controle negativo $(\mathrm{p}<0,05)$.

Alguns estudos apresentam somente a terapia de luz (irradiação sem a presença de um Fs) como um protocolo antimicrobiano alternativo a TFD. Contudo, quando comparado a genotoxicidade dos protocolos da terapia somente com luz LED $\left(89.2 \mathrm{~mW} / \mathrm{cm}^{2}\right.$ e $\left.440-460 \mathrm{~nm}\right)$ e da luz LED mediada por curcumina sobre $C$. albicans, foi constatado que, a primeira, produziu danos ao DNA das células fúngicas que foram rapidamente reparados ao passo que, a TFDa, produziu danos extensivos e irreversíveis. A curcumina inibe o reparo de danos ao material genético das células fúngicas e este pode ser o principal mecanismo de ação desta terapia (Carmello et al., 2015). Em nosso estudo, podemos verificar que, independente do protocolo de irradiação, apenas a aplicação da luz (grupos LED) não produziu redução do biofilme de nenhuma espécie de Candida, sendo necessário o emprego do produto vegetal para atuação contra a comunidade microbiana.

Quando avaliado o potencial da C. longa como Fs, a grande maioria dos estudos relatados na literatura falam somente do fitoquimico isolado: curcumina. Tais estudos apresentam excelentes resultados (Andrade et al., 2013; Lívia N. Dovigo et al., 2011; Lívia Nordi Dovigo et al., 2013). Contudo, de posse do conhecimento sobre o potencial da curcumina para mediar TFDa, os autores acreditaram ser importante estudar o potencial fototóxico do extrato de cúrcuma, uma vez que, a efetividade dos extratos naturais muitas vezes resulta a partir do efeito sinérgico de vários componentes (Iqbal Ahmad, Farrukh Aqil, 2006). Enquanto o composto principal pode ter ação em um ou mais alvos, outras moléculas do extrato podem atuar em outros alvos ou influenciar a cinética de absorção por exemplo (Gilbert \& Alves, 2005). No presente estudo, encontramos que a TFDa com extrato vegetal atuou de maneira eficiente contra biofilme de Candida spp. Estudos na literatura com a aplicação do extrato de C. Longa na TFDa mostraram que o extrato alcóolico foi eficaz como Fs sobre o periodontopatógeno Aggregatibacter actinomycetemcomitans produzindo efeito antimicrobiano equivalente a clorexidina $0,12 \%$ (Saitawee et al., 2018).

Contudo, o emprego da TFDa consiste na aplicação de um Fs não-tóxico associado a luz e em nossos achados, observamos que o produto vegetal possui potencial antifúngico quando aplicado isoladamente. Portanto, os autores acreditam que variações nas concentrações do extrato vegetal possam possibilitar novos caminhos a serem estudados como: o aumento da concentração do extrato para avaliar a ação antifúngica isolada e a diminuição da concentração para ser aplicado como Fs em diferentes protocolos de TFDa.

Uma vez que a candidose oral provoca uma resposta inflamatória no tecido das mucosas, encontrar um protocolo capaz de associar ação antimicrobiana com ação anti-inflamatória pode ser promissor. O protocolo de luz vermelha pulsada aplicada no presente trabalho consiste em uma variação bem interessante, uma vez que dentre as suas vantagens podemos citar: aceleração do processo de cicatrização de feridas, poder de analgesia e ação anti-inflamatória (Vieira et al., 2020). Os autores acreditam que tais vantagens são relevantes para seguir com os estudos in vivo e os achados do presente trabalho trazem contribuições importantes sobre o potencial antifúngico de terapias alternativas a serem aplicadas na odontologia. O extrato de C. longa apresenta potencial para ser explorado na fitoterapia e na fotoquimioterapia no comprimento de onda de $660 \mathrm{~nm}$, contra biofilme de Candida spp. A descoberta de sua potente ação antifúngica enriquece as possibilidades de alternativas terapêuticas no combate a candidose e demais infecções bucais na odontologia. 


\section{Conclusão}

O extrato de $C$. longa associado ou não a TFDa apresentou ação antibiofilme contra todas as cepas de Candida avaliadas e sua ação antifúngica foi igual e até superior a nistatina sobre algumas espécies. Para estudos futuros, os autores acreditam que explorar o potencial do extrato vegetal e o potencial das variações do protocolo de TFDa com luz vermelha em modelos animais in vivo possibilitará os avanços necessários em direção a descoberta de um protocolo terapêutico.

\section{Agradecimentos}

Ao Programa Institucional de Bolsas de Iniciação Cientifica (PIBIC) pela concessão da Bolsa de Iniciação Científica (Projeto PIBIC no 831 - vigência 01/09/2020 a 31/08/2021).

\section{Referências}

Andrade, M. C., Ribeiro, A. P. D., Dovigo, L. N., Brunetti, I. L., Giampaolo, E. T., Bagnato, V. S., \& Pavarina, A. C. (2013). Effect of different pre-irradiation times on curcumin-mediated photodynamic therapy against planktonic cultures and biofilms of Candida spp. Archives of Oral Biology, 58(2), 200-210. https://doi.org/10.1016/j.archoralbio.2012.10.011

Arastehfar, A., Carvalho, A., Hong Nguyen, M., Hedayati, M. T., Netea, M. G., Perlin, D. S., \& Hoenigl, M. (2020). Covid-19-associated candidiasis (Cac): An underestimated complication in the absence of immunological predispositions? In Journal of Fungi (Vol. 6, Issue 4, pp. 1-13). Multidisciplinary Digital Publishing Institute. https://doi.org/10.3390/jof6040211

Bruschi, M. L., da Silva, J. B., \& Rosseto, H. C. (2019). Photodynamic Therapy of Psoriasis Using Photosensitizers of Vegetable Origin. Current Pharmaceutical Design, 25(20), 2279-2291. https://doi.org/10.2174/1381612825666190618122024

Carmello, J. C., Pavarina, A. C., Oliveira, R., \& Johansson, B. (2015). Genotoxic effect of photodynamic therapy mediated by curcumin on Candida albicans. FEMS Yeast Research, 15(4), 18. https://doi.org/10.1093/femsyr/fov018

Cheraghipour, K., Ezatpour, B., Masoori, L., Marzban, A., Sepahvand, A., Rouzbahani, A. K., Moridnia, A., Khanizadeh, S., \& Mahmoudvand, H. (2020). Anti-Candida Activity of Curcumin: A Systematic Review. Current Drug Discovery Technologies, 18(3), 379-390. https://doi.org/10.2174/1570163817666200518074629

da Silva, F. C., Fernandes Rodrigues, P. L., Santos Dantas Araújo, T., Sousa Santos, M., de Oliveira, J. M., Pereira Rosa, L., de Oliveira Santos, G. P., de Araújo, B. P., \& Bagnato, V. S. (2019). Fluorescence spectroscopy of Candida albicans biofilms in bone cavities treated with photodynamic therapy using blue LED (450 nm)and curcumin. Photodiagnosis and Photodynamic Therapy, 26, 366-370. https://doi.org/10.1016/j.pdpdt.2019.05.002

Dovigo, Lívia N., Pavarina, A. C., Ribeiro, A. P. D., Brunetti, I. L., Costa, C. A. D. S., Jacomassi, D. P., Bagnato, V. S., \& Kurachi, C. (2011). Investigation of the photodynamic effects of curcumin against Candida albicans. Photochemistry and Photobiology, 87(4), 895-903. https://doi.org/10.1111/j.17511097.2011.00937.x

Dovigo, Lívia Nordi, Carmello, J. C., De Souza Costa, C. A., Vergani, C. E., Brunetti, I. L., Bagnato, V. S., \& Pavarina, A. C. (2013). Curcumin-mediated photodynamic inactivation of Candida albicans in a murine model of oral candidiasis. Medical Mycology, 51(3), 243-251. https://doi.org/10.3109/13693786.2012.714081

Gilbert, B., \& Alves, L. (2005). Synergy in Plant Medicines. Current Medicinal Chemistry, 10(1), 13-20. https://doi.org/10.2174/0929867033368583

Guandalini Cunha, B., Duque, C., Sampaio Caiaffa, K., Massunari, L., Araguê Catanoze, I., dos Santos, D. M., de Oliveira, S. H. P., \& Guiotti, A. M. (2020). Cytotoxicity and antimicrobial effects of citronella oil (Cymbopogon nardus) and commercial mouthwashes on S. aureus and C. albicans biofilms in prosthetic materials. Archives of Oral Biology, 109. https://doi.org/10.1016/J.ARCHORALBIO.2019.104577

Iqbal Ahmad, Farrukh Aqil, M. O. (2006). Turning Medicinal Plants into Drugs, Wiley VCH Verlag. Modern Phytomedicine.

Jerônimo, L. S., Esteves Lima, R. P., Suzuki, T. Y. U., Discacciati, J. A. C., \& Bhering, C. L. B. (2021). Oral Candidiasis and COVID-19 in Users of Removable Dentures: Is Special Oral Care Needed? Gerontology, 68(1), 1-6. https://doi.org/10.1159/000515214

Kawsud, P., Puripattanavong, J., \& Teanpaisan, R. (2014). Screening for anticandidal and antibiofilm activity of some herbs in Thailand. Tropical Journal of Pharmaceutical Research, 13(9), 1495-1501. https://doi.org/10.4314/tjpr.v13i9.16

Lee, Y., Puumala, E., Robbins, N., \& Cowen, L. E. (2021). Antifungal Drug Resistance: Molecular Mechanisms in Candida albicans and Beyond. Chemical Reviews, 121(6), 3390-3411. https://doi.org/10.1021/ACS.CHEMREV.0C00199

Li, X., Yin, L., Ramage, G., Li, B., Tao, Y., Zhi, Q., Lin, H., \& Zhou, Y. (2019). Assessing the impact of curcumin on dual-species biofilms formed by Streptococcus mutans and Candida albicans. MicrobiologyOpen, 8(12). https://doi.org/10.1002/mbo3.937

Monroy-Pérez, E., Rodríguez-Bedolla, R. M., Garzón, J., Vaca-Paniagua, F., Arturo-Rojas Jiménez, E., \& Paniagua-Contreras, G. L. (2020). Marked virulence and azole resistance in Candida albicans isolated from patients with periodontal disease. Microbial Pathogenesis, 148. https://doi.org/10.1016/J.MICPATH.2020.104436

Morio, F., Jensen, R. H., Le Pape, P., \& Arendrup, M. C. (2017). Molecular basis of antifungal drug resistance in yeasts. International Journal of 
Antimicrobial Agents, 50(5), 599-606. https://doi.org/10.1016/j.ijantimicag.2017.05.012

Nardini, E. F., Almeida, T. S., Yoshimura, T. M., Ribeiro, M. S., Cardoso, R. J., \& Garcez, A. S. (2019). The potential of commercially available phytotherapeutic compounds as new photosensitizers for dental antimicrobial PDT: A photochemical and photobiological in vitro study. Photodiagnosis and Photodynamic Therapy, 27, 248-254. https://doi.org/10.1016/j.pdpdt.2019.05.027

Pfaller, M. A., Diekema, D. J., Gibbs, D. L., Newell, V. A., Ellis, D., Tullio, V., Rodloff, A., Fu, W., \& Ling, T. A. (2010). Results from the artemis disk global antifungal surveillance study, 1997 to 2007: A 10.5-year analysis of susceptibilities of candida species to fluconazole and voriconazole as determined by CLSI standardized disk diffusion. Journal of Clinical Microbiology, 48(4), 1366-1377. https://doi.org/10.1128/JCM.02117-09

Pfaller, Michael A., Messer, S. A., Moet, G. J., Jones, R. N., \& Castanheira, M. (2011). Candida bloodstream infections: Comparison of species distribution and resistance to echinocandin and azole antifungal agents in Intensive Care Unit (ICU) and non-ICU settings in the SENTRY Antimicrobial Surveillance Program (2008-2009). International Journal of Antimicrobial Agents, 38(1), 65-69. https://doi.org/10.1016/j.ijantimicag.2011.02.016

Puspitawati, R., Maira, U., Suniarti, D. F., \& Salma, A. (2019). Inhibition and eradication effect of javanese turmeric (Curcuma xanthorrhiza roxb.) extract against mature phase biofilm of Candida albicans. Pesquisa Brasileira Em Odontopediatria e Clinica Integrada, 19(1), 4886. https://doi.org/10.4034/PBOCI.2019.191.89

Saitawee, D., Teerakapong, A., Morales, N. P., Jitprasertwong, P., \& Hormdee, D. (2018). Photodynamic therapy of Curcuma long a extract stimulated with blue light against Aggregatibacter actinomycetemcomitans. Photodiagnosis and Photodynamic Therapy, 22, 101-105. https://doi.org/10.1016/j.pdpdt.2018.03.001

Salerno, C., Pascale, M., Contaldo, M., Esposito, V., Busciolano, M., Milillo, L., Guida, A., Petruzzi, M., \& Serpico, R. (2011). Candida-associated denture stomatitis. Medicina Oral, Patologia Oral y Cirugia Bucal, 16(2). https://doi.org/10.4317/medoral.16.e139

Samadi, F. M., Suhail, S., Sonam, M., Sharma, N., Singh, S., Gupta, S., Dobhal, A., \& Pradhan, H. (2019). Antifungal efficacy of herbs. Journal of Oral Biology and Craniofacial Research, 9(1), 28-32. https://doi.org/10.1016/j.jobcr.2018.06.002

Sampaio, L. S., de Annunzio, S. R., de Freitas, L. M., Dantas, L. O., de Boni, L., Donatoni, M. C., de Oliveira, K. T., \& Fontana, C. R. (2020). Influence of light intensity and irradiation mode on methylene blue, chlorin-e6 and curcumin-mediated photodynamic therapy against Enterococcus faecalis. Photodiagnosis and Photodynamic Therapy, 31. https://doi.org/10.1016/J.PDPDT.2020.101925

Sueth-Santiago, V., Mendes-Silva, G. P., Decoté-Ricardo, D., \& De Lima, M. E. F. (2015). Curcumin, the golden powder from turmeric: Insights into chemical and biological activities. In Quimica Nova. 38(4), 538-552. https://doi.org/10.5935/0100-4042.20150035

Vieira, A. B. H. (2020). Os efeitos fisiológicos do led vermelho no tegumento. Revista Científica de Estética e Cosmetologia, 1(1), $28-38$.

Vieira, J. N., Gonçalves, C. L., Villarreal, J. P. V., Gonçalves, V. M., Lund, R. G., Freitag, R. A., Silva, A. F., \& Nascente, P. S. (2019). Chemical composition of essential oils from the apiaceae family, cytotoxicity, and their antifungal activity in vitro against candida species from oral cavity. Brazilian Journal of Biology, 79(3), 432-437. https://doi.org/10.1590/1519-6984.182206

Wainwright, M., Maisch, T., Nonell, S., Plaetzer, K., Almeida, A., Tegos, G. P., \& Hamblin, M. R. (2017). Photoantimicrobials—are we afraid of the light? In The Lancet Infectious Diseases. 17(2), e49-e55. https://doi.org/10.1016/S1473-3099(16)30268-7 